Bull. Korean Math. Soc. 51 (2014), No. 3, pp. 803-812

http://dx.doi.org/10.4134/BKMS.2014.51.3.803

\title{
HARMONIC MAPPINGS RELATED TO FUNCTIONS WITH BOUNDED BOUNDARY ROTATION AND NORM OF THE PRE-SCHWARZIAN DERIVATIVE
}

\author{
Staniseawa Kanas and Dominika KlimeK-SmęT
}

\begin{abstract}
Let $\mathcal{S}_{\mathcal{H}}^{0}$ be the class of normalized univalent harmonic mappings in the unit disk. A subclass $\mathcal{V}^{\mathcal{H}}(k)$ of $\mathcal{S}_{\mathcal{H}}^{0}$, whose analytic part is function with bounded boundary rotation, is introduced. Some bounds for functionals, specially harmonic pre-Schwarzian derivative, described in $\mathcal{V}^{\mathcal{H}}(k)$ are given.
\end{abstract}

\section{Introduction}

A harmonic mapping $f$ of the simply connected region $\Omega$ is a complex-valued function of the form

$$
f=h+\bar{g},
$$

where $h$ and $g$ are analytic in $\Omega$, with $g\left(z_{0}\right)=0$ for some prescribed point $z_{0} \in \Omega$. We call $h$ and $g$ analytic and co-analytic parts of $f$, respectively. If $f$ is (locally) injective, then $f$ is called (locally) univalent. The Jacobian and second complex dilatation of $f$ are given by $J_{f}(z)=\left|f_{z}\right|^{2}-\left|f_{\bar{z}}\right|^{2}=\left|h^{\prime}(z)\right|^{2}-\left|g^{\prime}(z)\right|^{2}$ and $\omega(z)=g^{\prime}(z) / h^{\prime}(z)(z \in \Omega)$, respectively. A result of Lewy [18] states that $f$ is locally univalent if and only if its Jacobian is never zero, and is sense-preserving if the Jacobian is positive. The sense-preserving case implies $|\omega(z)|<1$ in $\mathbb{D}$.

Throughout this paper we will assume that $f$ is locally univalent, sensepreserving, and $\Omega=\mathbb{D} \subset \mathbb{C}$, with $z_{0}=0$, where $\mathbb{D}$ is the open unit disk on the complex plane. Following Clunie and Sheil-Small notation [6], the class of all sense-preserving univalent harmonic mappings of $\mathbb{D}$ with $h(0)=g(0)=$ $h^{\prime}(0)-1=0$ we denote $\mathcal{S}_{\mathcal{H}}$, and its subclass for which $g^{\prime}(0)=0$ by $\mathcal{S}_{\mathcal{H}}^{0}$. Several fundamental information about harmonic mappings in the plane can be found in e.g. [8]. We note that each $f$ satisfying (1.1) in $\mathbb{D}$ is uniquely determined by

Received April 16, 2013.

2010 Mathematics Subject Classification. Primary 31A05; Secondary 30C45.

Key words and phrases. univalent harmonic mappings, functions with bounded boundary rotation. 
coefficients of the following power series expansions

$$
h(z)=\sum_{n=0}^{\infty} a_{n} z^{n}, \quad g(z)=\sum_{n=1}^{\infty} b_{n} z^{n} \quad(z \in \mathbb{D}),
$$

where $a_{n} \in \mathbb{C}, n=0,1,2, \ldots$ and $b_{n} \in \mathbb{C}, n=1,2,3, \ldots$. Moreover, when $f \in \mathcal{S}_{\mathcal{H}}$ we have $a_{0}=0, a_{1}=1$. In the sequel, we assume also $g^{\prime}(0)=b_{1}$ with $\left|b_{1}\right|=\alpha$. Taking into account the condition $|\omega(z)|<1$, immediately obtains $0 \leq \alpha<1$. Let

$$
\omega(z)=c_{0}+c_{1} z+c_{2} z^{2}+\cdots \quad(z \in \mathbb{D}) .
$$

According to the relation $\omega=g^{\prime} / h^{\prime}$ we have $c_{0}=b_{1}$ with $\left|b_{1}\right|=\alpha$. Therefore, we have

$$
\frac{|r-\alpha|}{1-\alpha r} \leq|\omega(z)| \leq \frac{r+\alpha}{1+\alpha r}
$$

and

$$
\left|c_{n}\right| \leq 1-\left|c_{0}\right|^{2}, \quad\left|\omega^{\prime}(z)\right| \leq \frac{1-|\omega(z)|^{2}}{1-|z|^{2}} \quad(z \in \mathbb{D}) .
$$

see e.g. $[4$, p. 30,53$]$.

Let $\mathcal{V}(k)$ denote the class of bounded boundary rotation, i.e., a class of normalized functions $f$ such that

$$
\int_{0}^{2 \pi}\left|\operatorname{Re}\left(1+\frac{r e^{i \theta} f^{\prime \prime}\left(r e^{i \theta}\right.}{f^{\prime}\left(r e^{i \theta}\right.}\right)\right| d \theta \leq k \pi,
$$

see [17]. We note that $\mathcal{V}\left(k_{1}\right) \subset \mathcal{V}\left(k_{2}\right)$ for $k_{1}<k_{2}$. We assume $k \geq 2$ and if $k \leq 4$, then $f$ is close-to-convex (the converse is not true). Let

$$
\begin{aligned}
F_{k}(z) & =\frac{1}{k}\left[\left(\frac{1+z}{1-z}\right)^{k / 2}-1\right]=\sum_{n=1}^{\infty} B_{n}(k) z^{n} \\
& =z+\frac{k}{2} z^{2}+\frac{k^{2}+2}{6} z^{3}+\cdots,
\end{aligned}
$$

$z \in \mathbb{D}$. Then the following estimates holds.

Theorem 1.1 ([11], t.II, pp. 16-25). If $f \in \mathcal{V}(k)$, then

$$
|f(z)| \leq F_{k}(r), \quad F_{k}^{\prime}(-r) \leq\left|f^{\prime}(z)\right| \leq F_{k}^{\prime}(r),
$$

and

$$
\left|a_{n}\right| \leq B_{n}(k) .
$$

In the present paper we introduce the concept of planar harmonic mappings with the analytic part being a function with bounded boundary rotation.

Definition 1.2. By $\mathcal{V}^{\mathcal{H}}(k)$ we denote the subclass of $\mathcal{S}_{\mathcal{H}}$ consisting of all harmonic mappings of the form $f=h+\bar{g}$ for which $h \in \mathcal{V}(k)$, with normalization $h(0)=g(0)=h^{\prime}(0)-1=0$ and $g^{\prime}(0)=b_{1},\left|b_{1}\right|=\alpha$. 
The classes of functions $f=h+\bar{g}$ with fixed analytic part were studied previously in the literature. We remain, for example, papers [13], [14], [15]; in [14] authors studied properties of a subset $\overline{\mathcal{S}}_{\mathcal{H}}^{\alpha}$ of $\mathcal{S}_{\mathcal{H}}$ consisting of all univalent anti-analytic perturbations of the identity whereas in [15] the class $\widehat{\mathcal{S}}^{\alpha}$ of all $f \in \mathcal{S}_{\mathcal{H}}$, such that $h$ is convex, normalized univalent functions.

\section{Coefficient and distortion results}

Theorem 2.1. Let $f$ be of the form (1.1) with the Taylor expansions (1.2) and $f \in \mathcal{V}^{\mathcal{H}}(k)$. Then

$$
\left|b_{n}\right| \leq \alpha B_{n}(k)+\frac{1-\alpha^{2}}{n} \sum_{p=1}^{n-1} p B_{p}(k)
$$

where $B_{n}(k)$ are given by (1.7). Specially, we have

$$
\left|b_{2}\right| \leq \frac{1-\alpha^{2}+\alpha k}{2}, \quad\left|b_{3}\right| \leq \frac{\left(1-\alpha^{2}\right)(1+k)}{2}+\frac{\alpha\left(k^{2}+2\right)}{6} .
$$

The result is sharp only for the case $n=2$.

Proof. By the relation $g^{\prime}=\omega h^{\prime}$ we have

$$
n b_{n}=\sum_{p=0}^{n-1}(p+1) a_{p+1} c_{n-p-1}=a_{n} c_{0}+\sum_{p=1}^{n-1} p a_{p} c_{n-p} .
$$

Observing that $c_{0}=b_{1}$ so that $\left|c_{0}\right|=\left|b_{1}\right|=\alpha$, and making use of (1.5), and $\left|a_{n}\right| \leq B_{n}(k)$ the assertion immediately follows. We note, that the bounds in (2.1) is sharp only for the case $n=2$. Indeed, defining $\omega(z)=\alpha+(1-\alpha) z$, $h(z)=F_{k}(z)$, and applying the relation $g^{\prime}=\omega h^{\prime}$ with $g(0)=0$ by integration we have

$$
g(z)=\alpha F_{k}(z)+(1-\alpha) z F_{k}(z)-(1-\alpha) \int_{0}^{z} F_{k}(w) d w .
$$

In a such case $b_{2}=g^{\prime \prime}(0) / 2=\left(1-\alpha^{2}+\alpha k\right) / 2$, that realizes equality for $b_{2}$ in (2.2).

Remark 2.1. The reasoning used in a proof of Theorem 2.1 may be applied to the bounds of coefficients of any harmonic functions $f=h+\bar{g}$, with an assumption $\left|g^{\prime}(0)\right|=\alpha$ and such that the coefficients of the analytic part $h$ satisfy $\left|a_{n}\right| \leq B_{n}$ for $n \geq 1$ (here $h(z)=z+a_{2} z^{2}+\cdots$ ). Such approach is also presented in [12].

Theorem 2.2. Let $f \in \mathcal{V}^{\mathcal{H}}(k)$. Then

$$
\frac{|r-\alpha|}{(1-\alpha r)\left(1-r^{2}\right)}\left(\frac{1-r}{1+r}\right)^{k / 2} \leq\left|g^{\prime}(z)\right| \leq \frac{(r+\alpha)}{(1+\alpha r)\left(1-r^{2}\right)}\left(\frac{1+r}{1-r}\right)^{k / 2}
$$




$$
\begin{aligned}
& |g(z)| \leq \frac{\alpha+r}{k(1+\alpha r)}\left(\frac{1-r}{1+r}\right)^{k / 2}-\frac{1-\alpha^{2}}{k} \int_{0}^{r}\left(\frac{1+t}{1-t}\right)^{k / 2} \frac{d r}{(1-\alpha t)^{2}}, \\
& |g(z)| \geq\left|\frac{\alpha-r}{k(1-\alpha r)}\left(\frac{1-r}{1+r}\right)^{k / 2}+\frac{1-\alpha^{2}}{k} \int_{0}^{r}\left(\frac{1-t}{1+t}\right)^{k / 2} \frac{d t}{(1-\alpha t)^{2}}\right|,
\end{aligned}
$$

$$
|f(z)| \leq F_{k}(r)+\frac{\alpha+r}{k(1+\alpha r)}\left(\frac{1-r}{1+r}\right)^{k / 2}-\frac{1-\alpha^{2}}{k} \int_{0}^{r}\left(\frac{1+t}{1-t}\right)^{k / 2} \frac{d r}{(1-\alpha t)^{2}} .
$$

Proof. By the relation $g^{\prime}=\omega h^{\prime}$ we have $\left|g^{\prime}(z)\right|=|\omega(z)|\left|h^{\prime}(z)\right|$. The assertion (2.4) now follows by (1.4) and (1.8).

We note that if $\varphi$ is univalent, and $m^{\prime}(r) \leq\left|\varphi^{\prime}(z)\right| \leq M^{\prime}(r)(0 \leq|z|=r<1)$ then $\int_{0}^{r} m^{\prime}(r) d r \leq|\varphi(z)| \leq \int_{0}^{r} M^{\prime}(r) d r$. Applying this together with (2.4) we obtain (2.5) and (2.6).

Remark 2.2. The bounds in (2.5), (2.6) and (2.7) may be represented by the Appell hypergeometric function $F_{1}\left(a ; b_{1}, b_{2} ; c ; x, y\right)$, of two real variables $x$ and $y$, when we apply the following

$$
\begin{aligned}
& \int_{0}^{r}\left(\frac{1+t}{1-t}\right)^{k / 2} \frac{d t}{(1-\alpha t)^{2}} \\
= & \frac{(-1)^{k / 2}}{\alpha}\left[\frac{1}{1-\alpha r} F_{1}\left(1 ; \frac{k}{2}, \frac{-k}{2}, 2 ; \frac{1-\alpha}{1-\alpha r}, \frac{1+\alpha}{1-\alpha r}\right)-F_{1}\left(1 ; \frac{k}{2}, \frac{-k}{2}, 2 ; 1-\alpha, 1+\alpha\right)\right] .
\end{aligned}
$$

Remark 2.3. For the special case $k=1$ we recover from Theorem 2.2 the distortion theorem for the harmonic mappings with analytic part being convex and univalent function (compare [15]).

\section{Pre-Schwarzian derivative}

The Schwarzian $S_{f}$ and pre-Schwarzian $T_{f}$ derivatives of a holomorphic and locally univalent function $f$ is defined by

$$
S_{f}=\left(\frac{f^{\prime \prime}}{f^{\prime}}\right)^{\prime}-\frac{1}{2}\left(\frac{f^{\prime \prime}}{f^{\prime}}\right)^{2}=\left(T_{f}\right)^{\prime}-\left(T_{f}\right)^{2} / 2 .
$$

The Schwarzian derivative is a basic tool in complex analysis; it measures the deviation of $f$ from a Möbius transformation. The hyperbolic sup-norm of $S_{f}$ $\left(T_{f}\right.$, respectively) is introduced as follows

$$
\left\|S_{f}\right\|=\sup _{z \in \mathbb{D}}\left(1-|z|^{2}\right)^{2}\left|S_{f}(z)\right|, \quad|| T_{f} \|=\sup _{z \in \mathbb{D}}\left(1-|z|^{2}\right)\left|T_{f}(z)\right| .
$$

Both, the Schwarzian and pre-Schwarzian play a central role in the theory of Teichmüller spaces, inner radius of univalence, quasiconformal extension, etc. We quote here one of the most important results. 
Theorem 3.1 (Ahlfors-Weill [1], see also Krauss [16], Nehari [19], Duren and Lehto [9]). Let $f$ be the function normalized and analytic in the unit disk. If $f$ is univalent, then $\left\|S_{f}\right\| \leq 6$. Conversely, if $\left\|S_{f}\right\| \leq 2$, then $f$ is univalent. Moreover, let $0 \leq k<1$. If $f$ extends to a $k$-quasiconformal mapping of the Riemann sphere $\overline{\mathbb{C}}$ then $\left\|S_{f}\right\| \leq 6 k$. Conversely, if $\left\|S_{f}\right\| \leq 2 k$, then $f$ extends to a $k$-quasiconformal mapping of $\overline{\mathbb{C}}$.

The first problem in the theory of locally univalent harmonic mappings, is to find a suitable definition of the Schwarzian derivative (the pre-Schwarzian derivative, respectively). A natural definition, using the differential geometry of associated minimal surface, has been proposed by Chuaqui, Duren and Osgood [5], and described by the formula

$$
S_{f}=2 \frac{\partial^{2}(\log \lambda)}{\partial z^{2}}-2\left(\frac{\partial(\log \lambda)}{\partial z}\right)^{2},
$$

where $\lambda=\left|h^{\prime}\right|+\left|g^{\prime}\right|$. In the case, when $f$ is analytic, $\lambda=\left|f^{\prime}\right|$, so that $\log \lambda=\log f^{\prime} / 2+\log \bar{f}^{\prime} / 2$, therefore (3.2) agrees with the classical formula (3.1). In connection with harmonic Schwarzian derivative we define harmonic pre-Schwarzian as

$$
T_{f}=\frac{2 \partial(\log \lambda)}{\partial z}
$$

which, in the analytic case becomes $f^{\prime \prime} / f^{\prime}$, as in (3.1).

We observe that, if $g^{\prime}=\omega h^{\prime}$, then [8, p. 191]

$$
S_{f}=S_{h}+\frac{2 \bar{\omega}}{1+|\omega|^{2}}\left(\omega^{\prime \prime}-\frac{\omega^{\prime} h^{\prime \prime}}{h^{\prime}}\right)-4\left(\frac{\omega^{\prime} \bar{\omega}}{1+|\omega|^{2}}\right)^{2},
$$

and

$$
T_{f}=\frac{2 \partial(\log \lambda)}{\partial z}=\frac{h^{\prime \prime}}{h^{\prime}}+\frac{2 \omega^{\prime} \bar{\omega}}{1+|\omega|^{2}}=T_{h}+\frac{2 \omega^{\prime} \bar{\omega}}{1+|\omega|^{2}} .
$$

Also, note that

$$
S_{f \circ \varphi}=\left(S_{f} \circ \varphi\right) \varphi^{\prime 2}+S_{\varphi}, \quad T_{f \circ \varphi}=T_{f} \circ \varphi+T_{\varphi} .
$$

The above formulas are generalization of the classical transformation formula for Schwarzian and pre-Schwarzian under composition.

In this section we find bounds of the norm of pre-Schwarzian derivative for co-analytic part of harmonic mapping from $\mathcal{S}_{\mathcal{H}}^{\alpha}$ and $\mathcal{V}^{\mathcal{H}}(k)$.

Before we prove the next theorems we remain some fact about the cardinals of polynomials roots. The best known is the classical rule of Descartes-Harriot, but it is not sufficient for computing the number of roots over a given interval. This problem was solved by Sturm, however less known but efficient method was presented by Vincent [20], using continued fractions. The modified Vincent's theorem e.g. its bisection version due to Alesina and Galuzzi [2] was presented after almost 200 years, in 2000. This method was next implemented by Vincent-Akritas-Strzeboński [3] and the continued fractions method for the 
determining the real zeros turns out to be the fastest method derived from Vincent's theorem.

Theorem 3.2 (Vincent, [20], bisection version (2000), [2], [3])). Let $p(x)$ be a polynomial of degree $n$. There exists a positive quantity $\delta$ so that for every pair of positive rational numbers $a, b$ with $|b-a|<\delta$ every transformed polynomial of the form

$$
V(x)=(1+x)^{n} p\left(\frac{a+b x}{1+x}\right)
$$

has exactly 0 or 1 variations in the sequence of its coefficients. The second case is possible if and only if $p(x)$ has a simple root within $(a, b)$. Moreover, the number of the sign variation is the maximal number of roots in $(a, b)$.

Theorem 3.3. The norm of the harmonic pre-Schwarzian derivative in the class $\mathcal{S}_{\mathcal{H}}^{\alpha}$ is bounded by

$$
\left\|T_{f}\right\| \leq 2\left(1-\alpha^{2}\right) \frac{\left(1-r_{0}^{2}\right)\left(\alpha+r_{0}\right)}{\left(1+\alpha r_{0}\right)\left[\left(1+\alpha^{2}\right)\left(1+r_{0}^{2}\right)-4 \alpha r_{0}\right]},
$$

where $r_{0}$ is the only root from the interval $(0,1)$ of the equation

$r_{0}^{4}\left(\alpha^{4}+4 \alpha^{2}-1\right)+4 \alpha r_{0}^{3}\left(1-\alpha^{2}\right)-4 r_{0}^{2}\left(\alpha^{4}+1\right)+4 r_{0} \alpha\left(\alpha^{2}-1\right)+1+4 \alpha^{2}-\alpha^{4}=0$.

Proof. Since $h(z) \equiv z$ then $T_{h} \equiv 0$ so that

$$
T_{f}=\frac{2 \omega^{\prime} \bar{\omega}}{1+|\omega|^{2}}
$$

Making use estimates (1.4), (1.5), we obtain for $|z|=r<1$

$$
\left|T_{f}\right| \leq \frac{2\left(1-\alpha^{2}\right)(\alpha+r)}{(1+\alpha r)\left[\left(1+\alpha^{2}\right)\left(1+r^{2}\right)-4 \alpha r\right]},
$$

so that

$$
\left\|T_{f}\right\| \leq 2\left(1-\alpha^{2}\right) \sup _{0<r<1} \frac{\left(1-r^{2}\right)(\alpha+r)}{(1+\alpha r)\left[\left(1+\alpha^{2}\right)\left(1+r^{2}\right)-4 \alpha r\right]} .
$$

The derivative of the function

$$
G(r):=\frac{\left(1-r^{2}\right)(\alpha+r)}{(1+\alpha r)\left[\left(1+\alpha^{2}\right)\left(1+r^{2}\right)-4 \alpha r\right]}
$$

is zero, if the function $H$, given by

$H(r):=r^{4}\left(\alpha^{4}+4 \alpha^{2}-1\right)+4 \alpha r^{3}\left(1-\alpha^{2}\right)-4 r^{2}\left(\alpha^{4}+1\right)+4 r \alpha\left(\alpha^{2}-1\right)+1+4 \alpha^{2}-\alpha^{4}$ takes its zero for $0<r<1$. Note, that $H(0)=1+4 \alpha^{2}-\alpha^{4}>0$ and $H\left(1^{-}\right)=-4\left(\alpha^{2}-1\right)^{2}<0$ then there exists $r_{0} \in(0,1)$ such, that $H\left(r_{0}\right)=0$. We prove the such root of $H$ is unique on $(0,1)$. It is enough to prove that the derivative

$$
H^{\prime}(r)=4 r^{3}\left(\alpha^{4}+4 \alpha^{2}-1\right)+12 \alpha r^{2}\left(1-\alpha^{2}\right)-8 r\left(\alpha^{4}+1\right)+4 \alpha\left(\alpha^{2}-1\right)
$$


is negative for $0<r<1$ and $0<\alpha<1$. Fix now $r$, and let $L(\alpha)=H^{\prime}(r)$ for $0<\alpha<1$ and $0<r<1$, so that

$L(\alpha)=\left(-8 r+4 r^{3}\right) \alpha^{4}+\left(4-12 r^{2}\right) \alpha^{3}+16 r^{3} \alpha^{2}+\left(-4+12 r^{2}\right) \alpha+\left(-8 r-4 r^{3}\right)$.

We note that $L(0)=-8 r-4 r^{3}$ and $L(1)=16 r\left(r^{2}-1\right)<0$. Next, define

$$
V(x)=(1+x)^{4} L\left(\frac{x}{1+x}\right) .
$$

Then, we have

$$
\begin{aligned}
V(x)= & -4 r\left(2+r^{2}\right)-4\left(1+8 r-3 r^{2}+4 r^{3}\right) x \\
& -4\left(1+8 r-3 r^{2}+4 r^{3}\right) x^{2} \\
& -8(1-r)\left(1+5 r+2 r^{2}\right) x^{3}-16 r\left(1-r^{2}\right) x^{4} .
\end{aligned}
$$

It is easy to check that the sign of the sequence of coefficient of $V(x)$ has the form $(-,-,-,-,-)$. Therefore there is no sign variation on $(0,1)$ for every $r \in(0,1)$. By the Vincent theorem we then conclude that there is no zeros of polynomial $L(\alpha)$ in the interval $(0,1)$. Since the function $L$ start from the negative value $L(0)$, therefore it must be negative in the entire interval, that implies the negativity of $H^{\prime}$.

Hence, $H$ is decreasing for $0<r<1$ and $0<\alpha<1$, and the equation (3.7) has the only root on the interval $(0,1)$, which is the only maximum of $G$ on $(0,1)$.

Theorem 3.4. The norm of pre-Schwarzian derivative in the class $\mathcal{V}^{\mathcal{H}}(k)$ is bounded by

$$
\left\|T_{f}\right\| \leq k+2 r_{0}+\frac{2\left(1-\alpha^{2}\right)\left(\alpha+r_{0}\right)}{\left(1+\alpha r_{0}\right)\left[\left(1+\alpha^{2}\right)\left(1+r_{0}^{2}\right)-4 \alpha r_{0}\right]},
$$

where $r_{0}$ is the only root of the equation

(3.11) $2+5 \alpha^{2}-4 \alpha^{4}+\alpha^{6}-2 r \alpha\left(5-2 \alpha^{2}+\alpha^{4}\right)+r^{2}\left(-2+9 \alpha^{2}-16 \alpha^{4}+5 \alpha^{6}\right)$

$$
+16 r^{3} \alpha^{3}+r^{4} \alpha^{2}\left(-7+2 \alpha^{2}+\alpha^{4}\right)+r^{5}\left(2 \alpha-4 \alpha^{3}-6 \alpha^{5}\right)+r^{6}\left(\alpha+\alpha^{3}\right)^{2}
$$

on the interval $(0,1)$.

Proof. Since $h \in \mathcal{V}(k)$ then $\left|T_{h}\right| \leq(k+2 r) /\left(1-r^{2}\right),|z|=r<1$, and

Therefore

$$
\left|T_{f}\right|=\left|T_{h}+\frac{2 \omega^{\prime} \bar{\omega}}{1+|\omega|^{2}}\right| \leq\left|T_{h}\right|+\left|\frac{2 \omega^{\prime} \bar{\omega}}{1+|\omega|^{2}}\right| .
$$

$$
\left\|T_{f}\right\| \leq \sup _{0<r<1}\left(1-r^{2}\right)\left[\frac{k+2 r}{1-r^{2}}+\frac{2\left(1-\alpha^{2}\right)(\alpha+r)}{(1+\alpha r)\left[\left(1+\alpha^{2}\right)\left(1+r^{2}\right)-4 \alpha r\right]}\right] .
$$

The derivative of the right hand function is equal to zero, if

$$
\begin{aligned}
& 2+5 \alpha^{2}-4 \alpha^{4}+\alpha^{6}-2 r \alpha\left(5-2 \alpha^{2}+\alpha^{4}\right)+r^{2}\left(-2+9 \alpha^{2}-16 \alpha^{4}+5 \alpha^{6}\right) \\
& +16 r^{3} \alpha^{3}+r^{4} \alpha^{2}\left(-7+2 \alpha^{2}+\alpha^{4}\right)+r^{5}\left(2 \alpha-4 \alpha^{3}-6 \alpha^{5}\right)+r^{6}\left(\alpha+\alpha^{3}\right)^{2}=0
\end{aligned}
$$


for $r \in(0,1)$. Denote the last polynomial by $P(r)$. Then

$$
P(0)=2+5 \alpha^{2}-4 \alpha^{4}+\alpha^{6}=2+4 \alpha^{2}\left(1-\alpha^{2}\right)+\alpha^{2}+\alpha^{6}>0,
$$

and

$$
P(1)=-8 \alpha+8 \alpha^{2}+16 \alpha^{3}-16 \alpha^{4}-8 \alpha^{5}+8 \alpha^{6}=-8 \alpha(1-\alpha)\left(1-\alpha^{2}\right)^{2}<0,
$$

so that there exists $r_{0} \in(0,1)$, such that $P\left(r_{0}\right)=0$. It suffices to prove that $r_{0}$ is unique. To claim this we prove that $P^{\prime}<0$ for $r \in(0,1)$. Let $r \in(0,1)$ be now fixed, and denote by $Q(\alpha)$ the derivative $P^{\prime}(r)$, that is

$$
\begin{aligned}
Q(\alpha)= & -4 r-10\left(1-r^{4}\right) \alpha+2 r\left(9-14 r^{2}+3 r^{4}\right) \alpha^{2} \\
& +\left(4+48 r^{2}-20 r^{4}\right) \alpha^{3}+\left(-32 r+8 r^{3}+12 r^{5}\right) \alpha^{4} \\
& +\left(-2-30 r^{4}\right) \alpha^{5}+\left(10 r+4 r^{3}+6 r^{5}\right) \alpha^{6} .
\end{aligned}
$$

We have $Q(0)=-4 r<0$ and $Q(1)=8(r-1)^{3}\left(1+4 r+3 r^{2}\right)<0$.

Let $S_{0}$ be the number of sign variation in the sequence of coefficients of the polynomial $Q(\alpha)$. Denoting the coefficients of $Q(\alpha)$ by $a_{0}, \ldots, a_{6}$ we have

$$
\begin{aligned}
& a_{0}=-4 r<0, \\
& a_{1}=-10+10 r^{4}<0, \\
& a_{2}=2 r\left(9-14 r^{2}+3 r^{4}\right)<0, \text { or }>0, \\
& a_{3}=4+48 r^{2}-20 r^{4}>0, \\
& a_{4}=4 r\left(-8+2 r^{2}+3 r^{4}\right)<0, \\
& a_{5}=-2\left(1+15 r^{4}\right)<0, \\
& a_{6}=10 r+4 r^{3}+6 r^{5}>0 .
\end{aligned}
$$

Hence the sequence of the sign of the coefficients $a_{i}(i=0, \ldots, 6)$ is $(-,-, \pm,+$, $-,-,+)$, so that the number of sign changes is $S_{0}=3$ for any $r \in(0,1)$. It means, by the classical rule of Descartes-Harriot, that there are 3 or 1 positive roots of $Q(\alpha)$. In order to show that there is no zero in $(0,1)$ we use the Vincent Theorem. The function

$$
V(\alpha)=(1+\alpha)^{6} Q\left(\frac{\alpha}{1+\alpha}\right)
$$

has the following coefficients

$$
\begin{aligned}
& b_{0}=-4 r<0, \\
& b_{1}=-10\left(1-r^{4}\right)-24 r<0, \\
& b_{2}=-2\left[\left(1-r^{4}\right)(25+3 r)+18 r+14 r^{3}\right]<0, \\
& b_{3}=-8\left[3\left(1-r^{2}\right)\left(2+r^{3}\right)+10 r^{3}(1-r 1)+6+r+r^{3}\right]<0, \\
& b_{4}=8(r-1)\left(2+9 r+9\left(1-r^{2}\right)+11 r^{3}+6 r^{4}\right)<0, \\
& b_{5}=-8(1-r)\left[1+4\left(1-r^{2}\right)+7 r(1-r)+r^{3}+6 r^{4}\right]<0,
\end{aligned}
$$




$$
b_{6}=8(r-1)^{3}(1+r)(1+3 r)<0,
$$

that form the following sequence of $\operatorname{sign}(-,-,-,-,-,-)$ with no sign variations. Thus, by the Vincent Theorem, there are no zeros at $(0,1)$ for any $r \in(0,1)$. It means that $Q(\alpha)<0$ for every $\alpha \in(0,1)$ and $r \in(0,1)$, equivalently $P^{\prime}(r)<0$ in $(0,1)$ that ends the proof.

Acknowledgements. This work was partially supported by the Centre for Innovation and Transfer of Natural Sciences and Engineering Knowledge.

\section{References}

[1] L. V. Ahlfors and G. Weill, A uniqueness theorem for Beltrami equations, Proc. Amer. Math. Soc. 13 (1962), 975-978.

[2] A. Alesina and M. Galuzzi, Vincent's theorem from a modern point of view, In R. Betti and W. F. Lawvere (Eds.), Categorical Studies in Italy 2000, Rend. Circ. Mat. Palermo, Serie II 64 (2000), 179-191.

[3] A. G. Akritas, A. W. Strzeboński, and P. S. Vigklas, Improving the performance of the continued fractions method using new bounds of positive roots, Nonlinear Anal. Model. Control 13 (2008), no. 3, 265-279.

[4] F. G. Avkhadiev and K. J. Wirths, Schwarz-Pick Type Inequalities, Birkhäuser Verlag AG, Basel-Boston-Berlin, 2009.

[5] M. Chuaqui, P. Duren, and B. Osgood, The Schwarzian derivative for harmonic mappings, J. Anal. Math. 91 (2003), 329-351.

[6] J. G. Clunie and T. Sheil-Small, Harmonic Univalent Functions, Ann. Acad. Sci. Fenn. Ser. Math. 9 (1984), 3-25.

[7] F. Colonna, The Bloch constant of bounded harmonic mappings, Indiana Univ. Math. J. 38 (1989), no. 4, 829-840.

[8] P. L. Duren, Harmonic Mappings in the Plane, Cambridge Tracts in Mathematics 156, Cambridge Univ. Press, Cambridge, 2004.

[9] P. L. Duren and O. Lehto, Schwarzian derivatives and homeomorphic extensions, Ann. Acad. Sci. Fenn. Ser. 477 (1970), 11 pp.

[10] J. Fourier, Analyse des équations déterminées, F. Didot, Paris, 1831.

[11] A. W. Goodman, Univalent Functions, Mariner Publishing, Tampa, 1983.

[12] D. Kalaj, S. Ponnusamy, and M. Vuorinen, Radius of close-to-convexity of harmonic functions, Complex Var. Elliptic Equ. (2013), to appear.(arXiv:1107.0610)

[13] S. Kanas and D. Klimek, Coefficient estimates and Bloch's constant in some class of harmonic mappings, to appear.

[14] D. Klimek and A. Michalski, Univalent anti-analytic perturbations of the identity in the unit disc, Sci. Bull. Chełm 1 (2006), 67-76.

[15] - Univalent anti-analytic perturbations of convex analytic mappings in the unit disc, Ann. Univ. Mariae Curie-Skłodowska Sect. A Vol. LXI (2007), Sectio A, 39-49.

[16] W. Krauss, Über den Zusammenhang einiger Clarakteristiken eines einfach zusammenhängenden Bereiches mit der Kreisabbildung, Mitt. Math. Sem. Giessen 21 (1932), $1-28$.

[17] O. Lehto, On the distortion of conformal mappings with bounded boundary rotation, Ann. Acad. Sci. Fenn. Ser. Math. 124 (1952), no. 124, 14pp.

[18] H. Lewy, On the non-vanishing of the Jacobian in certain one-to-one mappings, Bull. Amer. Math. Soc. 42 (1936), no. 10, 689-692.

[19] Z. Nehari, The Schwarzian derivatives and schlicht functions, Bull. Amer. Math. Soc. 55 (1949), 545-551. 
[20] A. J. H. Vincent, Sur la résolution des équations numériques, J. Math. Pures Appl. 1 (1836), 341-372.

STANISEAWA KANAS

UNIVERSITY OF RZESZOW

Al. Rejtana 16c, 35-959 Rzeszow, Poland

E-mail address: stankanas@gmail.com

DOMINIKA KLIMEK-SMET

Maria Curie-SkŁodowska University

Pl. Marit Curie-Skeodowskiej 5, 20-031 Lublin, Poland

E-mail address: dominika@hektor.umcs.lublin.pl 